\title{
ОПРЕДЕЛЕНИЕ ЗНАЧИМЫХ ФАКТОРОВ ДЛЯ ПРОВЕДЕНИЯ ДИФФЕРЕНЦИАЛЬНОЙ ДИАГНОСТИКИ САХАРНОГО ДИАБЕТА 2 ТИПА И МОDY С ПОМОЩЬЮ БИНАРНОЙ ЛОГИСТИЧЕСКОЙ РЕГРЕССИИ
}

\author{
Овсянникова А.К., Шахтшнейдер Е.В., Иванощук Д.Е., Рымар О.Д.
}

\begin{abstract}
Научно-исследовательский институт терапии и профилактической медицины — филиал Федерального государственного бюджетного научного учреждения «Федеральный исследовательский чентр Институт иитологии и генетики Сибирского отделения Российской академии наук», Новосибирск
\end{abstract}

В клинической практике врача эндокринолога очень важно своевременно диагностировать правильный тип сахарного диабета (СД) у пациентов молодого возраста, так как именно в этой возрастной группе может определяться сахарный диабет 1 типа, сахарный диабет 2 типа (СД2), LADA диабет и моногенные формы, в том числе, MODY. Верификация MODY довольно сложна, однако, она позволяет назначить патогенетическую терапию, обеспечить адекватное ведение беременности, дает возможность провести профилактику специфических для конкретного подтипа осложнений.

ЦЕЛЬ: определить значимые факторы для проведения дифференциальной диагностики СД2 и MODY.

МАТЕРИАЛЫ И МЕТОДЫ: в данное исследование включены 128 пациентов С диагностированным СД в возрасте с 18 до 35 лет: у 63 верифицирован молекулярно-генетическим исследованием (MГИ) MODY диабет, у 65 - СД2. МГИ выполнено с использованием технологии секвенирования следующего поколения и прямого автоматического секвенирования по Сэнгеру. Всем пациентам проведен клинический осмотр, биохимический анализ крови, определение $\mathrm{HbA}_{1 c^{\prime}}$ С-пептида, тиреоидного статуса, МАУ, УЗИ органов брюшной полости, щитовидной железы, эхокардиография, дуплексное сканирование брахиоцефальных сосудов на экстракраниальном уровне. Статистическая обработка результатов исследования проводилась на персональном компьютере с помощью программы SPSS 15. Связи между признаками оценивались вычислением коэффициента линейной корреляции Пирсона. Для интервальных и порядковых переменных, не подчиняющихся нормальному распределению - рангового коэффициента корреляции Спирмена. Для оценки зависимости клинических и лабораторных параметров от типа СД использовалась бинарная логистическая регрессия.

PEЗУЛЬTATЫ: группу пациентов с MODY (1 группа) составили 36 лиц с GCK-MODY, 16 - с HNF1A-MODY, 11 - с редкими типами MODY, группу с СД2 (2 группа) - 65 человек. Медиана возраста пациентов на момент осмотра была в 1 группе 24,0 [0,0;35,0] лет, во 2 - 29,5 [17,7;35,0] (p=0,280). Обе группы были сопоставимы по полу, возрасту и продолжительности СД.

Были проанализированы клинические, в том числе, наследственность, лабораторные показатели при диагностировании СД. Определены наиболее значимые признаки ( $<<0,001)$, ассоциированные с типом СД, которые использовались при создании модели бинарной логистической регресии. Качество модели получилось достаточно высоким, корректно сгруппированы 77\% наблюдений. Высоко достоверными были следующие факторы: наличие родственников с диагностированным СД в возрасте до 35 лет (ассоциировано с MODY), наличие родственников с СД и избыточным весом, избыточный вес у исследуемых пациентов (ассоциировано с СД2).

\section{Выводы:}

1. При проведении дифференциальной диагностики типа СД у лиц с дебютом заболевания до 35 лет необходимо учитывать наличие отягощенного семейного анамнеза по нарушениям углеводного обмена и избыточного веса.

2. Наличие у пациентов молодого возраста избыточного веса ассоциировано с СД2. 